\title{
Uncertainty principle of quantum processes
}

\author{
Yunlong Xiao $\odot,{ }^{1,2,3,4, *}$ Kuntal Sengupta $\odot,{ }^{3,4}$ Siren Yang, ${ }^{3,4}$ and Gilad Gour ${ }^{3,4, \dagger}$ \\ ${ }^{1}$ School of Physical and Mathematical Sciences, Nanyang Technological University, Singapore 639673, Singapore \\ ${ }^{2}$ Complexity Institute, Nanyang Technological University, Singapore 639673, Singapore \\ ${ }^{3}$ Department of Mathematics and Statistics, University of Calgary, Calgary, Alberta T2N 1N4, Canada \\ ${ }^{4}$ Institute for Quantum Science and Technology, University of Calgary, Calgary, Alberta, T2N 1N4, Canada
}

(Received 19 April 2020; accepted 29 March 2021; published 28 April 2021)

\begin{abstract}
Heisenberg's uncertainty principle, which imposes intrinsic restrictions on our ability to predict the outcomes of incompatible quantum measurements to arbitrary precision, demonstrates one of the key differences between classical and quantum mechanics. The physical systems considered in the uncertainty principle are static in nature and described mathematically with a quantum state in a Hilbert space. However, many physical systems are dynamic in nature and described with the formalism of a quantum channel. In this paper, we show that the uncertainty principle can be reformulated to include process measurements that are performed on quantum channels. Since both the preparation of quantum states and the implementation of quantum measurements are themselves special cases of quantum channels, our formalism encapsulates the uncertainty principle in its utmost generality. More specifically, we obtain expressions that generalize the Maassen-Uffink uncertainty relation and the universal uncertainty relations from quantum states to quantum channels.
\end{abstract}

DOI: 10.1103/PhysRevResearch.3.023077

\section{INTRODUCTION}

Counterintuitive as it may seem, the uncertainty principle has been firmly rooted as a fundamental restriction that lies at the heart of quantum mechanics [1]. The amount of information one can extract from a quantum system at any given time depends on the extent of the incompatibility of the underlying measurements involved. In Heisenberg's uncertainty principle, this corresponds to the fact that any attempt to measure the position of a quantum particle with very high precision comes at the cost of poor precision in the simultaneous measurement of its momentum. This fundamental distinction from classical physics has led to enormous research in the area and found a plethora of applications in quantum key distribution [2] and the detection of quantum resources [3], such as entanglement [4-10], Einstein-Podolsky-Rosen steering [11-17], and Bell nonlocality [18].

The uniqueness and immense potential in quantum uncertainty has caused Heisenberg's uncertainty principle-which was mathematically formulated by Kennard [19] (also refer to Weyl [20]) — to go through multiple refinements over the last century. One such example is the use of Rényi entropies to formulate uncertainty relations from an information-theoretic perspective by Maassen and Uffink [21] (based on the Riesz

\footnotetext{
*mathxiao123@gmail.com

$\dagger$ gour@ucalgary.ca

Published by the American Physical Society under the terms of the Creative Commons Attribution 4.0 International license. Further distribution of this work must maintain attribution to the author(s) and the published article's title, journal citation, and DOI.
}

theorem [22]):

$$
\mathrm{H}_{\alpha}(M)+\mathrm{H}_{\beta}(N) \geqslant-2 \log c(M, N) .
$$

Here, $\mathrm{H}_{\alpha}(M):=\frac{1}{1-\alpha} \log \left(\sum_{x} p_{x}^{\alpha}\right)$ stands for the Rényi entropy with order $\alpha>0$, where $\mathbf{p}=\left\{p_{x}\right\}$ is the probability vector corresponding to the outcomes of the measurement $M$ when performed on a system in a state $\rho$. The Rényi parameters $\alpha$ and $\beta$ are chosen such that $1 / \alpha+1 / \beta=2$. The constant $c(M, N)$ stands for the maximal overlap between the measurements $M$ and $N$ and is independent of the state $\rho$.

More recently, the authors of Ref. [23] showed that not only entropic functions, but any nonnegative Schur-concave function is a suitable uncertainty quantifier for the probabilities obtained from measurements, giving rise to a class of infinitely many uncertainty relations, known as universal uncertainty relations (UURs). It is critical to note that the classification of UURs with respect to the joint uncertainty they present is a major focus in the theory of uncertainty relations. While considering the two probability distributions $\mathbf{p}$ and $\mathbf{q}$ obtained by measuring quantum state $\rho$ with respect to measurements $M$ and $N$, their joint uncertainty can be based on direct product [23-25] as well as direct sum [26]

$$
\begin{gathered}
\mathbf{p} \otimes \mathbf{q} \prec \mathbf{b}_{\otimes}, \\
\mathbf{p} \oplus \mathbf{q} \prec \mathbf{b}_{\oplus} .
\end{gathered}
$$

Here, $\mathbf{b}_{\otimes}$ and $\mathbf{b}_{\oplus}$ are probability vectors independent of the initial state $\rho$, and " $\prec$ " stands for majorization (for $\mathbf{x}=$ $\left(x_{k}\right)_{k}, \mathbf{y}=\left(y_{k}\right)_{k} \in \mathbb{R}^{d}$, we have $\mathbf{x} \prec \mathbf{y}$ whenever $\sum_{k=1}^{i} x_{k}^{\downarrow} \leqslant$ $\sum_{k=1}^{i} y_{k}^{\downarrow}$ for all $1 \leqslant i \leqslant d-1$ and $\sum_{k=1}^{d} x_{k}=\sum_{k=1}^{d} y_{k}$, where the down arrow $\downarrow$ means the components of the corresponding 
vector are arranged in nonincreasing order). The approach of majorization adopted by Refs. [23,25] frees us from particular measures and captures the essence of uncertainty in quantum mechanics.

In quantum mechanics, the conventional description of the uncertainty principle captures the statistical properties of the system to be studied at a fixed moment, leading to the mathematical representation of uncertainty relations in terms of the initial state. However, this approach has serious insufficiencies when it comes to describing many realistic scenarios and hence has impeded progress in understanding the physical nature of the quantum world. Let us name a few shortcomings of this conventional description: First, everything is in motion in the universe, and the tendency of quantum states is to evolve. Thus, when formulating a quantitative expression of the tradeoff between incompatible measurements, we should not only focus on the static state at a fixed moment, but also the quantum process it undergoes. Second, the preparation of the quantum state and the implementation of quantum measurements are all special cases of quantum processes which occur in a real laboratory. Nature owns more plentiful and important quantum processes, ranging from the dynamics of molecules in chemistry [27] to the functions of biochemical systems in biology [28] and non-Markovian quantum processes in physics [29-31]. Furthermore, even quantum artificial intelligence such as quantum machine learning [32], which might contain complex internal structures, is a dynamical quantum process, so it is necessary to investigate the amount of information one can have about quantum processes under incompatible process measurements. The case of the preparation of quantum states has been answered by Heisenberg's uncertainty principle, and a large number of subsequent works has been dedicated to its reformulation and improvements, but the answer to the general case of quantum processes is still absent.

In this paper, we extend the uncertainty principle to incorporate the case of quantum dynamical processes, generalizing the notion of quantum uncertainty to a theory of quantum mechanics modeled solely by quantum processes. We investigate a Rényi entropic uncertainty relation, generalizing the celebrated relation in Eq. (1) from quantum states to quantum processes, and present direct-sum and direct-product UURs for quantum processes which extend the UURs in Eqs. (2) and (3) to quantum processes. Examples that support our results have also been supplied.

\section{PRELIMINARIES}

In this section, we give a brief discussion of notation, terminology, and background information on the formulation of dynamical quantum processes. For a finite dimensional Hilbert space $\mathcal{H}$, the set of all linear transformation taking the Hilbert space to itself is denoted by $L(\mathcal{H})$. An operator $\rho \in L(\mathcal{H})$ is a density operator, representing a quantum state, if it is positive semidefinite and has unit trace, i.e., $\rho \geqslant 0$ and $\operatorname{Tr}[\rho]=1$. We denote the collection of all density operators on $\mathcal{H}$ as $D(\mathcal{H})$. A quantum effect $M_{x}$ on $D(\mathcal{H})$ is an operator such that $0 \leqslant M_{x} \leqslant \mathbb{1}$, where $\mathbb{1}$ denotes the identity matrix on $D(\mathcal{H})$. The probability $p_{x}$ of an outcome $x$ as a result of the effect $M_{x}$ acting on a density operator $\rho$ is given by $\operatorname{Tr}\left[M_{x} \rho\right]$.
A positive-operator-valued measure (POVM) $M=\left\{M_{x}\right\}_{x}$ is a set of effects that collectively sum to the identity $\sum_{x} M_{x}=\mathbb{1}$.

A superoperator $\Psi$ maps operators of one Hilbert space to operators of another Hilbert space. Naturally, for Hilbert spaces $\mathcal{H}^{A}$ and $\mathcal{H}^{B}, \Psi\left[L\left(\mathcal{H}^{A}\right)\right] \subset L\left(\mathcal{H}^{B}\right)$ or simply $\Psi: A \rightarrow$ $B$, and their collection is denoted by $T(A, B):=\{\Psi \mid \Psi: A \rightarrow$ $B$. A superoperator $\Psi \in T(A, B)$ is said to be a quantum channel if it is (i) completely positive $\left(\mathrm{CP}\right.$, i.e., $\mathbb{1}^{R} \otimes \Psi$ is positive for all finite dimensional Hilbert space $\mathcal{H}^{R}$ ), and (ii) trace preserving (TP, i.e., $\left.\operatorname{Tr}_{B}[\Psi(\bullet)]=\operatorname{Tr}_{A}[\bullet]\right)$. We use $\operatorname{CPTP}(A, B)$ to denote the collection of all CPTP maps from space $L\left(\mathcal{H}^{A}\right)$ to space $L\left(\mathcal{H}^{B}\right)$.

Now let us look at states and measurements from the perspective of quantum channels: a quantum state $\rho$ can be seen as the state-preparation channel $\Gamma_{\rho}: \mathbb{C} \rightarrow \mathcal{H}$, and a POVM $M=\left\{M_{x}\right\}_{x}$ is equivalent to the measurement channel $\Lambda_{M}$ : $\mathcal{H} \rightarrow \mathbb{C}$. To deal with dynamical quantum processes, i.e., CPTP maps, it is convenient to use the Choi-Jamiołkowski (CJ) isomorphism [33,34]:

Lemma 1 (Choi-Jamiotkowski). For any $\Psi \in T(A, B)$, there is a linear bijection between $T(A, B)$ and $L(A \otimes B):=$ $L\left(\mathcal{H}^{A} \otimes \mathcal{H}^{B}\right)$, which is given by

$$
\begin{aligned}
\theta: T(A, B) & \rightarrow L(A \otimes B), \\
\Psi & \mapsto J_{\Psi}^{A B},
\end{aligned}
$$

where $\quad J_{\Psi}^{A B}=\mathbb{1}^{\tilde{A} \rightarrow A} \otimes \mathbb{1}^{B}\left(J_{\Psi}^{\tilde{A} B}\right)=\mathbb{1}^{\tilde{A} \rightarrow A} \otimes \Psi\left(\phi_{+}^{\tilde{A} A}\right) \quad$ with $\phi_{+}^{\tilde{A A}}:=\left|\phi_{+}^{\tilde{A} A}\right\rangle\left\langle\phi_{+}^{\tilde{A} A}\right|$ being an unnormalized maximally entangled state with $\left|\phi_{+}^{\tilde{A} A}\right\rangle:=\sum_{i=1}^{d_{A}}|i\rangle^{\tilde{A}}|i\rangle^{A}$.

Here, the tilde symbol indicates an identical copy of the system under it, and hence, in what follows, we do not distinguish between the space $\mathcal{H}^{\tilde{A}}$ and $\mathcal{H}^{A}$. Analogous to the role of density operator of a quantum system, the CJ matrix $J_{\Psi}^{A B}$ provides a complete description of the physical process $\Psi$ at any given time.

The entire study of quantum information theory revolves around how much information can be efficiently packed, transferred, and retrieved in a desired fashion by means of preparation, manipulation, and measurement of quantum states [35-37]. In the theory of quantum mechanics modeled solely by quantum channels, this whole picture, therefore, boils down to the idea of storing and retrieving information, not from quantum states, but from quantum channels themselves. However, just like quantum states, the only way of accessing information from a quantum channel is by measuring it. Such a measurement is called process-channel measurement, introduced in Ref. [38]. Imagine a scenario with a state-preparation device providing initial state $\rho^{R A} \in$ $L(R \otimes A)$ and a POVM $M=\left\{M_{x}\right\}_{x}$ acting on $L(R \otimes B)$. Formally, the process-channel measurement is defined by the couple $\mathcal{T}:=\left(\rho^{R A}, M\right)$. To retrieve the information conveyed by the quantum channel $\Psi$, a reference system $R$ distributes to the measuring device directly; meanwhile, the probe system $A$, which is correlated with $R$, is transformed through $\Psi$ followed by the measurement $M$. Here, the classical information or measurement outcome $x$ will occur with probability $p_{x}=\operatorname{Tr}\left[M_{x} \mathbb{1}^{R} \otimes \Psi\left(\rho^{R A}\right)\right]$. Next, we substitute the equation $\rho^{R A}=\Upsilon_{\rho} \otimes \mathbb{1}^{A}\left(\phi_{+}^{\tilde{A} A}\right)$, where $\Upsilon_{\rho}: L\left(\mathcal{H}^{\tilde{A}}\right) \rightarrow L\left(\mathcal{H}^{R}\right)$ is a CP 
linear map [36,37], into the expression for $p_{x}$; we get

$$
\begin{aligned}
p_{x} & =\operatorname{Tr}\left[M_{x} \mathbb{1}^{R} \otimes \Psi\left(\rho^{R A}\right)\right] \\
& =\operatorname{Tr}\left\{M_{x} \mathbb{1}^{R} \otimes \Psi\left[\Upsilon_{\rho} \otimes \mathbb{1}^{A}\left(\phi_{+}^{\tilde{A A}}\right)\right]\right\} \\
& =\operatorname{Tr}\left[\Upsilon_{\rho}^{*} \otimes \mathbb{1}^{B}\left(M_{x}\right) \mathbb{1}^{A} \otimes \Psi\left(\phi_{+}^{\tilde{A A}}\right)\right] \\
& =\operatorname{Tr}\left[\Upsilon_{\rho}^{*} \otimes \mathbb{1}^{B}\left(M_{x}\right) J_{\Psi}^{A B}\right],
\end{aligned}
$$

where in the third equation, $\Upsilon_{\rho}^{*}$ is the dual map of $\Upsilon_{\rho}$, which is also a CP linear map, with the property that, for all operators $M^{A} \in L(A):=L\left(\mathcal{H}^{A}\right)$ and for all $M^{R} \in L(R):=L\left(\mathcal{H}^{R}\right)$, we have $\operatorname{Tr}\left\{\left[\Upsilon_{\rho}\left(M^{A}\right)\right]^{\dagger} M^{R}\right\}=\operatorname{Tr}\left[\left(M^{A}\right)^{\dagger} \Upsilon_{\rho}^{*}\left(M^{R}\right)\right]$.

Regarding the above discussions, it is clear that, for each single channel measurement $\left(\rho^{R A}, M_{x}\right)$, we can define an operator $E_{x}:=\Upsilon_{\rho}^{*} \otimes \mathbb{1}^{A}\left(M_{x}\right) \geqslant 0$ satisfying

$$
p_{x}=\operatorname{Tr}\left[E_{x} J_{\Psi}^{A B}\right] \text {. }
$$

Here, $E_{x}$ is the so-called process-channel effect of single channel measurement $\left(\rho^{R A}, M_{x}\right)$, and their collection $\left\{E_{x}\right\}_{x}$ is known as process POVM (PPVOM) or tester [38]. More generally, a PPOVM is a special case of 2 combs [39-41], where preprocessing and postprocessing are classical-to-quantum and quantum-to-classical channels, respectively.

\section{MAASSEN-UFFINK UNCERTAINTY RELATIONS}

Having defined what a measurement of quantum process is, we now use it to study entropic uncertainty relations. Let $\Psi$ be a quantum channel from operator space $L(A)$ to $L(B)$. For simplicity of the exposition, we start with two PPOVMs and denote them as $\mathcal{T}_{1}:=\left(\rho^{R A}, M\right)$ and $\mathcal{T}_{2}:=\left(\sigma^{R A}, N\right)$. We also denote by $\left\{p_{x}\right\}_{x}$ and $\left\{q_{y}\right\}_{y}$ the two probability distributions obtained by measuring $\Psi$ with respect to $\mathcal{T}_{1}$ and $\mathcal{T}_{2}$. In analogy with $E_{x}$ and $p_{x}$, let us also define $F_{y}=\Upsilon_{\sigma}^{*} \otimes \mathbb{1}^{A}\left(N_{y}\right) \geqslant 0$ as the process-channel effect of single channel measurement $\left(\sigma^{R A}, N_{y}\right)$, such that $q_{y}=\operatorname{Tr}\left(F_{y} J_{\Psi}^{A B}\right)$. It is straightforward to check that $\sum_{x} E_{x}=\left(\rho^{A}\right)^{\mathrm{T}} \otimes \mathbb{1}^{B} \leqslant \mathbb{1}^{A B}$ and $\sum_{y} F_{y}=\left(\sigma^{A}\right)^{\mathrm{T}} \otimes$ $\mathbb{1}^{B} \leqslant \mathbb{1}^{A B}$, where ${ }^{\mathrm{T}}$ denotes transposition in the corresponding space, and hence the mathematical structure of PPOVMs do not obey the completeness relation [38].

Next, we will introduce the overlap for PPOVMs by extending the sets of process-channel effect $\left\{E_{x}\right\}_{x=1}^{m}$ and $\left\{F_{y}\right\}_{y=1}^{n}$ to $\left\{\tilde{E}_{x}\right\}_{x=1}^{m+1}$ and $\left\{\tilde{F}_{y}\right\}_{y=1}^{n+1}$, respectively. Regarding the subscript, the extended process-channel effects $\tilde{E}_{x}, \tilde{F}_{y}$ are defined as

$$
\tilde{E}_{x}:=\left\{\begin{array}{cc}
E_{x} & 1 \leqslant x \leqslant m, \\
\mathbb{1}^{A B}-\left(\rho^{A}\right)^{\mathrm{T}} \otimes \mathbb{1}^{B} & x=m+1,
\end{array}\right.
$$

and

$$
\tilde{F}_{y}:=\left\{\begin{array}{cc}
F_{y} & 1 \leqslant y \leqslant n, \\
\mathbb{1}^{A B}-\left(\sigma^{A}\right)^{\mathrm{T}} \otimes \mathbb{1}^{B} & y=n+1 .
\end{array}\right.
$$

The quantity $c_{x y}\left(\mathcal{T}_{1}, \mathcal{T}_{2}\right):=\left\|\tilde{E}_{x}^{1 / 2} \tilde{F}_{y}^{1 / 2}\right\|$ with $1 \leqslant x \leqslant m+1$ and $1 \leqslant y \leqslant n+1$ represents the overlap between processchannel measurements $\mathcal{T}_{1}$ and $\mathcal{T}_{2}$, analogous to the overlap between projective measurements [42], extensively investigated in many quantum information-theory contexts, for example, Ref. [42]. The maximum overlap between $\mathcal{T}_{1}$ and $\mathcal{T}_{2}$ then can be defined as $c\left(\mathcal{T}_{1}, \mathcal{T}_{2}\right):=\max _{x, y} c_{x y}\left(\mathcal{T}_{1}, \mathcal{T}_{2}\right)$.

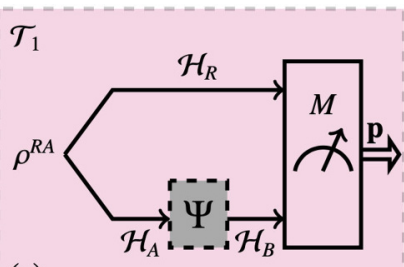

(a)

FIG. 1. Schematic illustration of the process positive-operatorvalued measures (PPOVMs): (a) PPOVM $\mathcal{T}_{1}$ and (b) PPOVM $\mathcal{T}_{2}$.

Guided by intuition, $c$ should provide a bound on the minimum uncertainty arising from simultaneously measuring $\Psi$ with $\mathcal{T}_{1}$ and $\mathcal{T}_{2}$, thereby quantifying the inherent incompatibility between process-channel measurements. We will return to the meaning of this later.

To establish our uncertainty relations, we collect the probabilities $p_{x}$ and $q_{y}$ into two probability vectors $\mathbf{p}:=$ $\mathbf{p}\left(\mathcal{T}_{1}, \Psi\right)=\left(p_{1}, \ldots, p_{m}\right)$ and $\mathbf{q}:=\mathbf{q}\left(\mathcal{T}_{2}, \Psi\right)=\left(q_{1}, \ldots, q_{n}\right)$, respectively (Fig. 1). Having defined what the probability vectors for process-channel measurements are, we now consider the corresponding uncertainty measure.

In classical information theory, entropy describes the uncertainty associated with a random variable and hence becomes a suitable candidate for uncertainty measure [42]. Inspired by Maassen and Uffink [21], we base our first result on the class of Rényi entropies defined as

$$
\mathrm{H}_{\alpha}(\mathbf{p}):=-\frac{1}{1-\alpha} \log \left(\sum_{x=1}^{m} p_{x}^{\alpha}\right),
$$

with $\alpha>0$ and $\alpha \neq 1$.

As with the primal formulation of Maassen-Uffink uncertainty relations [21], our first goal is to bound the joint uncertainty between $\mathbf{p}$ and $\mathbf{q}$ in terms of $\mathrm{H}_{\alpha}\left(\mathcal{T}_{1}\right)+\mathrm{H}_{\beta}\left(\mathcal{T}_{2}\right)$. This will turn out to be different from Ref. [43] by a quantity which depends only on the process effects $E_{x}$ and $F_{y}$ but not on the process $\Psi$ itself.

Theorem 2. For probability vectors $\mathbf{p}$ and $\mathbf{q}$ obtained by measuring $\Psi$ with respect to $\mathcal{T}_{1}:=\left(\rho^{R A}, M\right)$ and $\mathcal{T}_{2}:=$ $\left(\sigma^{R A}, N\right)$, their joint uncertainties in terms of $\mathrm{H}_{\alpha}\left(\mathcal{T}_{1}\right)+$ $\mathrm{H}_{\beta}\left(\mathcal{T}_{2}\right)$ is bounded by the maximum overlap $c\left(\mathcal{T}_{1}, \mathcal{T}_{2}\right)$ as

$$
\begin{aligned}
& \mathrm{H}_{\alpha}\left(\frac{1}{d_{A}} \mathbf{p} \oplus \frac{d_{A}-1}{d_{A}}\right)+\mathrm{H}_{\beta}\left(\frac{1}{d_{A}} \mathbf{q} \oplus \frac{d_{A}-1}{d_{A}}\right) \\
& \quad \geqslant-2 \log c\left(\mathcal{T}_{1}, \mathcal{T}_{2}\right),
\end{aligned}
$$

where $\alpha$ and $\beta$ satisfy the harmonic condition $1 / \alpha+1 / \beta=2$.

The left-hand side of Eq. (10) relies on the initial state of the quantum process $\Psi$ and the incompatible process-channel measurements $\mathcal{T}_{1}, \mathcal{T}_{2}$. The right-hand side is an irreducible bound for its joint uncertainty, which depends only on the incompatible process-channel measurements $\mathcal{T}_{1}, \mathcal{T}_{2}$ and can be calculated explicitly. With the help of Eq. (10), we can also derive the Shannon entropic uncertainty relation for quantum processes by taking limits of $\alpha$ and $\beta$ to approach 1 .

It is interesting to remark that, for a state-preparation channel $\Gamma_{\rho}$, we have $d_{A}=\operatorname{dim} \mathbb{C}=1$ for any state $\rho$, and hence, $\frac{1}{d_{A}} \mathbf{p} \oplus \frac{d_{A}-1}{d_{A}}=\mathbf{p}, \frac{1}{d_{A}} \mathbf{q} \oplus \frac{d_{A}-1}{d_{A}}=\mathbf{q}$. Additionally, the PPOVMs will degenerate into POVMs, and the maximum 
overlap $c\left(\mathcal{T}_{1}, \mathcal{T}_{2}\right)$ reduces to $c(M, N)$ [44]. This shows why our Thm. 2 includes the Maassen-Uffink uncertainty relation as a special case. Moreover, note that the bound described by Eq. (10) is tight since, for the case with $\alpha=\infty$ or $\beta=\infty$, $-2 \log c\left(\mathcal{T}_{1}, \mathcal{T}_{2}\right)$ is achieved by some quantum process [45]. We sketch the proof of our Thm. 2 in Appendix A.

\section{UURS}

We now turn our attention to the UURs for quantum processes. Traditionally, entropies like $\mathrm{H}_{\alpha}$ have been employed to study the uncertainty of probability distribution associated with measurements. However, in Ref. [23], the authors showed that the notion of majorization can fully characterize the uncertainty related with probability distributions and therefore capture the "the essence of uncertainty in quantum mechanics." Another motivation for the considerations of majorization uncertainty relations is that majorization, as a preorder, is more informative than the ones based on particular uncertainty measures, such as Shannon or Rényi entropies. Here, we will see that the joint distributions $\mathbf{p} \otimes \mathbf{q}$ and $\mathbf{p} \oplus \mathbf{q}$ obtained by measuring quantum processes are bounded by vectors independent of $\Psi$.

Let us first collect all process effects from $\mathcal{T}_{1}, \mathcal{T}_{2}$ together, and define their collections as

$$
G_{z}:=\left\{\begin{array}{lc}
E_{z} & 1 \leqslant z \leqslant m, \\
F_{z-m} & m+1 \leqslant z \leqslant m+n .
\end{array}\right.
$$

It follows that the general experiments measuring the quantum process $\Psi$ with $\mathcal{T}_{1}$ and $\mathcal{T}_{2}$ are completely characterized by the set of process effects $G$. For a subset $\mathcal{I}_{k} \subset\{1, \ldots, m+n\}$ with cardinality $k$, we define $G\left(\mathcal{I}_{k}\right):=\sum_{z \in \mathcal{I}_{k}} G_{z}$. With these conventions, the second goal of our paper is to bound the joint uncertainty in the form of $\mathbf{p} \oplus \mathbf{q}$. More precisely,

Theorem 3. For probability vectors $\mathbf{p}$ and $\mathbf{q}$ obtained by measuring $\Psi$ with respect to $\mathcal{T}_{1}:=\left(\rho^{R A}, M\right)$ and $\mathcal{T}_{2}:=$ $\left(\sigma^{R A}, N\right)$, their joint uncertainties in terms of $\mathbf{p} \oplus \mathbf{q}$ is bounded by a vector independent of quantum process $\Psi$ of the form

$$
\mathbf{p} \oplus \mathbf{q} \prec \mathbf{s}:=\left(s_{1}, s_{2}-s_{1}, s_{3}-s_{2}, \ldots, 0\right),
$$

where each $s_{k}$ is a functional of the conditional min-entropy

$$
s_{k}:=\max _{\mathcal{I}_{k}} 2^{-\mathrm{H}_{\min }(B \mid A)_{G\left(I_{k}\right)},}
$$

and the maximization is over all subsets $\mathcal{I}_{k}$. The conditional min-entropy for $G\left(\mathcal{I}_{k}\right)$ is defined as

$$
\mathrm{H}_{\min }(B \mid A)_{G\left(\mathcal{I}_{k}\right)}:=-\log \inf _{X^{A} \geqslant 0}\left[\operatorname{Tr}\left(X^{A}\right) \mid X^{A} \otimes \mathbb{1}^{B} \geqslant G\left(\mathcal{I}_{k}\right)\right] .
$$

Note that the operator $G\left(\mathcal{I}_{k}\right)$ is a process-channel effect, which is also an unnormalized quantum state. Thus, the conditional min-entropy defined above is not our usually used one for bipartite states. To find a formula based on the well-known conditional min-entropy, we can define a bipartite quantum state as $\tau\left(\mathcal{I}_{k}\right)=G\left(\mathcal{I}_{k}\right) / \operatorname{Tr}\left[G\left(\mathcal{I}_{k}\right)\right] \in D(A \otimes B)$, which depends on the subset $\mathcal{I}_{k}$, and call it a process-channel state corresponding to $G\left(\mathcal{I}_{k}\right)$. Consequently, $\mathrm{H}_{\min }(B \mid A)_{\tau^{A B}\left(I_{k}\right)}$ is the conditional min-entropy of the bipartite state $\tau^{A B}\left(\mathcal{I}_{k}\right)$. Now the quantity $s_{k}$ can be expressed as

$$
s_{k}=\max _{I_{k}} 2^{\left\{-\mathrm{H}_{\min }(B \mid A)_{\tau\left(I_{k}\right)}+\log \operatorname{Tr}\left[G\left(I_{k}\right)\right]\right\}} .
$$

We remark that the tightness of $s_{k}$ and the rigorous proof of Thm. 3 are detailed in Appendix B.

Aside from its numerous applications in single-shot quantum information, quantum hypothesis testing, and quantum resource theories, we show that this entropic quantifier has operational significance in terms of the tightness of the UURs for quantum processes with direct-sum form, which might also have an impact on the development of future technologies of quantum processes.

Continuing our discussion of UURs for quantum processes, we now show that the joint uncertainty based on the direct product, i.e., $\mathbf{p} \otimes \mathbf{q}$, can also be similarly characterized as stated in Thm. 4, whose proof is given in Appendix D.

Theorem 4. For probability vectors $\mathbf{p}$ and $\mathbf{q}$ obtained by measuring $\Psi$ with respect to $\mathcal{T}_{1}:=\left(\rho^{R A}, M\right)$ and $\mathcal{T}_{2}:=$ $\left(\sigma^{R A}, N\right)$, their joint uncertainties in terms of $\mathbf{p} \otimes \mathbf{q}$ is therefore bounded by a vector independent of quantum process $\Psi$ of the form

$$
\mathbf{p} \otimes \mathbf{q} \prec \mathbf{t}:=\left(t_{1}, t_{2}-t_{1}, t_{3}-t_{2}, \ldots, 0\right),
$$

with $t_{k}$ is defined by $\left(s_{k+1} / 2\right)^{2}$ constructed in Thm. 3 .

We finish by remarking that the class of Schur-concave functions can preserve the preorder induced by majorization; that is, for a Schur-concave function $\Phi: \mathbb{R}^{d} \rightarrow \mathbb{R}$ and $x$, $y \in \mathbb{R}^{d}, \Phi(\mathbf{x}) \geqslant \Phi(\mathbf{y})$ whenever $\mathbf{x} \prec \mathbf{y}$. As a result, the UURs for quantum processes in terms of $\mathbf{p} \oplus \mathbf{q} \prec \mathbf{s}$ and $\mathbf{p} \otimes \mathbf{q} \prec \mathbf{t}$ generate an infinite family of uncertainty relations of the forms $\Phi(\mathbf{p} \oplus \mathbf{q}) \geqslant \Phi(\mathbf{s})$ and $\Phi(\mathbf{p} \otimes \mathbf{q}) \geqslant \Phi(\mathbf{t})$ with each $\Phi$. Taking $\Phi$ as Shannon entropy H, Eqs. (12) and (16) will lead to the Shannon entropic uncertainty relations for quantum processes $\mathrm{H}\left(\mathcal{T}_{1}\right)+\mathrm{H}\left(\mathcal{T}_{2}\right) \geqslant \mathrm{H}(\mathbf{s})$ and $\mathrm{H}\left(\mathcal{T}_{1}\right)+\mathrm{H}\left(\mathcal{T}_{2}\right) \geqslant \mathrm{H}(\mathbf{t})$ with $\mathrm{H}\left(\mathcal{T}_{1}\right):=\mathrm{H}(\mathbf{p})$ and $\mathrm{H}\left(\mathcal{T}_{2}\right):=\mathrm{H}(\mathbf{q})$. However, the result presented in Thm. 2 is not covered by UURs, since in Eq. (10), the uncertainty associated with $\mathbf{p}$ and $\mathbf{q}$ is quantified by different uncertainty measures.

\section{CONCLUSIONS AND DISCUSSIONS}

In this paper, we have addressed the question of whether quantum mechanics will obstruct us from predicting the outcomes of incompatible process-channel measurements to arbitrary precision. We studied uncertainty relations in three distinct forms: Maassen-Uffink form; direct-sum form; and direct-product form, which reduces to the well-known Maassen-Uffink entropic uncertainty relations [21] and UURs $[23,25,26]$ as our special cases by choosing the process $\Psi$ to be a state-preparation channel $\Gamma_{\rho}$, i.e., $\Psi=\Gamma_{\rho}$.

Following Deutsch's observation [42], to express the uncertainty principle for quantum processes $\Psi: A \rightarrow B$ quantitatively, we are seeking an inequality with the form $\mathcal{U}\left(\mathcal{T}_{1}, \mathcal{T}_{2}, \Psi\right) \geqslant \mathcal{B}\left(\mathcal{T}_{1}, \mathcal{T}_{2}\right)$, where the quantity on the lefthand side represents the joint probability distribution induced by measuring quantum process $\Psi$ with PPOVMs $\mathcal{T}_{1}$ and $\mathcal{T}_{1}$ in the form of $\mathcal{U}$, with the optimal bound $\mathcal{B}\left(\mathcal{T}_{1}, \mathcal{T}_{2}\right):=\min _{\Psi \in \operatorname{CPTP}(A, B)} \mathcal{U}\left(\mathcal{T}_{1}, \mathcal{T}_{2}, \Psi\right)$. If we denote the set of all state-preparation channels as $\Gamma \subset \operatorname{CPTP}(A, B)$, the 
celebrated Heisenberg's uncertainty principle, with the form $\mathcal{U}\left(\mathcal{T}_{1}, \mathcal{T}_{2}, \Gamma_{\rho}\right) \geqslant \min _{\Psi \in \Gamma} \mathcal{U}\left(\mathcal{T}_{1}, \mathcal{T}_{2}, \Psi\right)$, becomes a special case of our generalized uncertainty principle.

Our first main result shows that the potential knowledge one can have about any quantum process from a pair of process-channel measurements $\mathcal{T}_{1}$ and $\mathcal{T}_{2}$, quantified by the Rényi entropies with harmonic condition, is restricted by their inherent incompatibility in terms of $c\left(\mathcal{T}_{1}, \mathcal{T}_{2}\right)$.

Secondly, we derived the UURs for quantum processes, i.e., Eqs. (12) and (16), which are the generalizations of the previous ones for quantum states and are explicitly computable. A natural question is whether the processindependent bounds $\mathbf{s}$ and $\mathbf{t}$ are optimal. For the sum of each $k$ distinct elements in $\mathbf{p} \oplus \mathbf{q}$, their upper-bound $s_{k}$ is tight, which means $s_{k}$ is achieved by performing $\mathcal{T}_{1}$ and $\mathcal{T}_{2}$ to some quantum processes. However, the vector $\mathbf{s}$ consisting of $s_{k}$ is not optimal. In Appendix C, we show that the optimal bound for $\mathbf{p} \oplus \mathbf{q}$ exists and is given by the vector $\mathcal{F}(\mathbf{s})$, where $\mathcal{F}$ stands for the flatness process [46]. On the other hand, even though the existence of the optimal bound $\mathbf{r}$ for $\mathbf{p} \otimes \mathbf{q}$ is guaranteed by the completeness of majorization lattice [47-50], so far we do not have any effective method for calculating it in general. Although the bound $\mathbf{t}$ introduced in Eq. (16) is weaker than $\mathbf{r}$, it is easy to evaluate. Like the method for the direct sum, the flatness process $\mathcal{F}$ can further improve the bound of the direct product to $\mathbf{p} \otimes \mathbf{q} \prec \mathbf{r} \prec \mathcal{F}(\mathbf{t}) \prec \mathbf{t}$. As a byproduct of UURs for quantum processes, we show that the optimal bound for the direct-sum form is specified completely by the conditional min-entropy, which connects UURs with single-shot information theory.

Finally, we address a number of interesting directions of future investigations, which have a close connection to the framework explored here, in Appendix E, and leave a conjecture on the Shannon entropic uncertainty relation for quantum processes in Appendix F.

\section{ACKNOWLEDGMENTS}

We would like to thank Eric Chitambar, Kun Fang, Li Gao, Mile Gu, Anna Jenčová, Nicholas LaRacuente, Zhihao Ma, Varun Narasimhachar, Carlo Maria Scandolo, Gaurav Saxena, Jayne Thompson, Kunkun Wang, Peng Xue, Lei Xiao, and Yuxiang Yang for fruitful discussions. Y.X. and G.G. acknowledge financial support from the Natural Sciences and Engineering Research Council of Canada (NSERC). Y.X. is supported by the National Research Foundation (NRF). Singapore, under its NRFF Fellow programme (Grant No. NRF-NRFF2016-02), Singapore Ministry of Education Tier 1 Grant No. MOE2017-T1-002-043 from the Foundational Questions Institute and Fetzer Franklin Fund Grant No. FQXi-RFP-1809 (a donor-advised fund of Silicon Valley Community Foundation). Any opinions, findings and conclusions or recommendations expressed in this material are those of the author(s) and do not reflect the views of National Research Foundation, Singapore.

\section{APPENDIX A: PROOF OF THEOREM 2}

In this section, we turn our attention to the MaassenUffink-form uncertainty relations for quantum processes. We will first briefly review the historical developments of the Maassen-Uffink uncertainty relation before formulating our generalized uncertainty principle in terms of Rényi entropies.

Deutsch introduced the uncertainty principle in terms of Shannon entropy for any two nondegenerate observables [42]. The improved bound on Deutsch uncertainty relation was conjectured by Kraus [51] and was proved by Maassen and Uffink [21]. The uncertainty measure adopted by Maassen and Uffink is Rényi entropy, an improvement over Shannon entropic uncertainty relations. The original result of Ref. [42] is only valid for pure states with Von Neumann measurements, and their proof relies on the Riesz theorem [22]. It is thus natural to ask whether the Maassen-Uffink uncertainty relation also holds for mixed states with POVMs, which was answered by Rastegin [44].

Lemma 5 (Rastegin). For probability vectors $\mathbf{p}$ and $\mathbf{q}$ obtained by measuring quantum state $\rho$ with respect to POVMs $M$ and $N$, their joint uncertainties in terms of $\mathrm{H}_{\alpha}(M)+\mathrm{H}_{\beta}(N)$ is therefore bounded by the maximum overlap $c(M, N, \rho)$ of the form

$$
\mathrm{H}_{\alpha}(M)+\mathrm{H}_{\beta}(N) \geqslant-2 \log c(M, N, \rho),
$$

where $\alpha$ and $\beta$ satisfy the harmonic condition $1 / \alpha+1 / \beta=2$. Here, the quantity $c(M, N, \rho)$ is defined by

$$
c(M, N, \rho):=\max _{\rho=\sum_{k} u_{k}\left|u_{k}\right\rangle\left\langle u_{k}\right|} \max _{x, y} \frac{\operatorname{Tr}\left(M_{x}^{\dagger} N_{y}\left|u_{k}\right\rangle\left\langle u_{k}\right|\right)}{\| M_{x}^{1 / 2}\left|u_{k}\right\rangle\|\cdot\| N_{y}^{1 / 2}\left|u_{k}\right\rangle \|} .
$$

The method of proof employed Naimark's dilation theorem [52] and the Riesz theorem as expected. By using the properties of operator norm, that is $\|\bullet\|:=\max \{\| \bullet$ $u\|\mid\| u \|=1$ \}, lemma 5 leads to the following entropic uncertainty relations with a state-independent bound $c(M, N):=$ $\max _{x, y}\left\|M_{x}^{1 / 2} N_{y}^{1 / 2}\right\|$ :

Corollary 6 (Rastegin). For probability vectors $\mathbf{p}$ and $\mathbf{q}$ obtained by measuring quantum state $\rho$ with respect to POVMs $M$ and $N$, their joint uncertainties in terms of $\mathrm{H}_{\alpha}(M)+\mathrm{H}_{\beta}(N)$ are therefore bounded by the maximum overlap $c(M, N)$ of the form

$$
\mathrm{H}_{\alpha}(M)+\mathrm{H}_{\beta}(N) \geqslant-2 \log c(M, N),
$$

where $\alpha$ and $\beta$ satisfy the harmonic condition $1 / \alpha+1 / \beta=2$.

There are two ways of proving the Maassen-Uffink uncertainty relation for quantum processes. The first one is to apply Naimark's dilation theorem to the $\mathrm{CJ}$ matrix $J_{\Psi}^{A B}$ with respect to the process $\Psi$, followed by the Riesz theorem. Another way is to use corollary 6 directly, which has been adopted here.

For probability distribution $\mathbf{p}$ specified by the processchannel measurement $\mathcal{T}_{1}$, the probability associated with measurement outcome $x$, as shown in Eq. (6) of our main text, is $p_{x}=\operatorname{Tr}\left(E_{x} J_{\Psi}^{A B}\right)$, and hence

$$
\frac{p_{x}}{d_{A}}=\operatorname{Tr}\left(E_{x} \rho_{\Psi}^{A B}\right),
$$

with $\rho_{\Psi}^{A B}:=J_{\Psi}^{A B} / d_{A}$ being a bipartite quantum state in $D(A \otimes$ $B$ ), since $\rho_{\Psi}^{A B} \geqslant 0$ (due to the CP of $\Psi$ ) and $\operatorname{Tr}\left(\rho_{\Psi}^{A B}\right)=1$ (due to the TP of $\Psi$ ). Therefore, the probability distribution $\frac{1}{d_{A}} \mathbf{p} \oplus$ $\frac{d_{A}-1}{d_{A}}$ can be seen as derived by performing POVM $\left\{\tilde{E}_{x}\right\}_{x=1}^{m+1}$ to 
the state $\rho_{\Psi}^{A B}$. Consider also the probability distribution $\frac{1}{d_{A}} \mathbf{q} \oplus$ $\frac{d_{A}-1}{d_{A}}$ obtained by implementing POVM $\left\{\tilde{F}_{y}\right\}_{y=1}^{n+1}$ to $\rho_{\Psi}^{A B}$; then corollary 6 immediately implies that

$$
\mathrm{H}_{\alpha}(\tilde{E})+\mathrm{H}_{\beta}(\tilde{F}) \geqslant-2 \log c\left(\mathcal{T}_{1}, \mathcal{T}_{2}\right),
$$

with $1 / \alpha+1 / \beta=2$. Written in full, that is

$$
\begin{gathered}
\mathrm{H}_{\alpha}\left(\frac{1}{d_{A}} \mathbf{p} \oplus \frac{d_{A}-1}{d_{A}}\right)+\mathrm{H}_{\beta}\left(\frac{1}{d_{A}} \mathbf{q} \oplus \frac{d_{A}-1}{d_{A}}\right) \\
\geqslant-2 \log c\left(\mathcal{T}_{1}, \mathcal{T}_{2}\right),
\end{gathered}
$$

as required.

\section{APPENDIX B: PROOF OF THEOREM 3}

Our goal in this section is to prove Eq. (12) of our main text. Let us first consider the following question: for any semidefinite positive operator $W \in L(A \otimes B)$, what is the maximal value of $\operatorname{Tr}\left(W J_{\Psi}^{A B}\right)$ for all quantum processes? We are interested in

$$
\begin{array}{cc}
\max & \operatorname{Tr}\left(W J_{\Psi}^{A B}\right), \\
\text { s.t. } & \operatorname{Tr}_{B} J_{\Psi}^{A B}=\mathbb{1}^{A}, \\
& J_{\Psi}^{A B} \geqslant 0 .
\end{array}
$$

which is semidefinite programming (SDP). The Lagrangian associated to the primal SDP in (B1) is given by

$$
\begin{aligned}
\mathscr{L} & =\operatorname{Tr}\left(W J_{\Psi}^{A B}\right)+\operatorname{Tr}_{A}\left[X\left(\mathbb{1}^{A}-\operatorname{Tr}_{B} J_{\Psi}^{A B}\right)\right]+\operatorname{Tr}\left(Y J_{\Psi}^{A B}\right) \\
& =\operatorname{Tr}(X)+\operatorname{Tr}\left[\left(W+Y-X \otimes \mathbb{1}^{A}\right) J_{\Psi}^{A B}\right],
\end{aligned}
$$

where we have introduced dual variables, i.e., Lagrange multipliers $X$, a Hermitian operator acting on Hilbert space $\mathcal{H}^{A}$, and $Y$, a semidefinite positive operator acting on Hilbert space $\mathcal{H}^{A} \otimes \mathcal{H}^{B}$, to ensure that the Lagrangian $\mathscr{L}$ is always greater than the objective function whenever the primal constraints are satisfied. Therefore, in this case, the dual SDP is obtained by minimizing over all dual variables:

$$
\begin{array}{ll}
\min & \operatorname{Tr}(X), \\
\text { s.t. } & X \otimes \mathbb{1}^{B} \geqslant W,
\end{array}
$$

Here, the strong duality holds since the primal SDP is finite and strictly feasible, which guarantees that the optimal value of dual coincides with the optimal value of the primal problem. The optimal value is related with the conditional min-entropy mentioned in our main text. We now move to the definition of conditional min-entropy [53], which is the main object of study in this section.

Definition 7 (Min-entropy). Let $\rho \in D(A \otimes B)$ be a bipartite quantum operator. The min-entropy of $A$ conditioned on $B$ is defined by

$$
\mathrm{H}_{\min }(A \mid B)_{\rho}:=-\inf _{\sigma} \mathrm{D}_{\max }\left(\rho \| \mathbb{1}^{A} \otimes \sigma\right),
$$

where the infimum ranges over all semidefinite positive operator $\sigma \in L(B)$, with

$$
\mathrm{D}_{\max }(\tau \| \eta):=\inf \left\{\lambda \in \mathbb{R} \mid 2^{\lambda} \eta \geqslant \tau\right\} .
$$

Now it is clear from the context that the optimal value of Eq. (B3) $=2^{-\mathrm{H}_{\min }(B \mid A)_{W}}$, which is equivalent to say that, for any quantum process $\Psi: A \rightarrow B$, we have

$$
\max _{\Psi} \operatorname{Tr}\left[W J_{\Psi}^{A B}\right]=2^{-\mathrm{H}_{\min }(B \mid A)_{W}} .
$$

We now move on to discuss the sum of the first $k$ largest components of $\mathbf{p} \oplus \mathbf{q}$, i.e.,

$$
\begin{aligned}
& \max _{|R|+|S|=k} \max _{\Psi}\left(\sum_{x \in R} p_{x}+\sum_{y \in S} q_{y}\right) \\
& =\max _{\mathcal{I}_{k}} \max _{\Psi} \operatorname{Tr}\left[\left(\sum_{z \in I_{k}} G_{z}\right) J_{\Psi}^{A B}\right] \\
& =\max _{\mathcal{I}_{k}} \max _{\Psi} \operatorname{Tr}\left[G\left(\mathcal{I}_{k}\right) J_{\Psi}^{A B}\right] \\
& =\max _{\mathcal{I}_{k}} 2^{-\mathrm{H}_{\min }(B \mid A)_{G\left(I_{k}\right)}} \\
& =s_{k} .
\end{aligned}
$$

with $R \subset\{1, \ldots, n\}, S \subset\{1, \ldots, m\}$, and $|\bullet|$ stands for the cardinality of set $\bullet$. Here, to arrive at the third line, we used the result shown in Eq. (B6), and the last line follows from the definition of $s_{k}$. Noticing now that, when the first $k$ largest components of $\mathbf{p} \oplus \mathbf{q}$ are upper-bounded by the quantity $s_{k}$, the vector $\mathbf{p} \oplus \mathbf{q}$ is thus majorized by $\left(s_{1}, s_{2}-s_{1}, s_{3}-s_{2}, \ldots, 0\right)$. We finally remark that, for the sum of the first $k$ largest components, $s_{k}$ is tight for all $k$, since there always exists a quantum process, which might not be unique, such that $\max _{|R|+|S|=k}\left(\sum_{x \in R} p_{x}+\sum_{y \in S} q_{y}\right)=s_{k}$. Even though each $s_{k}$ is tight, their collection $\mathbf{s}$ is not always guaranteed to be optimal. The optimal bound for $\mathbf{p} \oplus \mathbf{q}$ will be given in the next section by considering the lattice structure of majorization.

\section{APPENDIX C: MAJORIZATION LATTICE}

In this section, we turn our attention to the concept of lattice and employ the majorization lattice to study the optimal bounds of UURs for quantum processes. For simplicity, all vectors considered in this section belong to the set $\mathbb{R}^{d}$. Let us start with the definition of Lattice, which is

Definition 8 (Lattice). A quadruple $(S, \sqsubset, \wedge, \vee)$ is called a lattice if $\sqsubset$ is a partial order on the set $S$ such that, for all $\mathbf{p}$, $\mathbf{q} \in S$, there exists a unique greatest lower bound (GLB) $\mathbf{p} \wedge \mathbf{q}$ and a unique least upper bound (LUB) $\mathbf{p} \vee \mathbf{q}$ satisfying

$$
\begin{aligned}
& \mathbf{x} \sqsubset \mathbf{p}, \mathbf{x} \sqsubset \mathbf{q} \Rightarrow \mathbf{x} \sqsubset \mathbf{p} \wedge \mathbf{q}, \\
& \mathbf{p} \sqsubset \mathbf{y}, \mathbf{q} \sqsubset \mathbf{y} \Rightarrow \mathbf{p} \vee \mathbf{q} \sqsubset \mathbf{y} .
\end{aligned}
$$

for each $\mathbf{x}, \mathbf{y} \in S$.

A special class of lattices are those which have GLB and LUB for all their subsets, namely complete lattice:

Definition 9 (Complete Lattice). A lattice $(S, \sqsubset, \wedge, \vee)$ is called complete if, for any nonempty subset $R \subset S$, it has a LUB, denoted by $\vee R$ and a GLB, denoted by $\wedge R$. More precisely, if $\mathbf{x}, \mathbf{y} \in S$ such that $\mathbf{x} \sqsubset R \sqsubset \mathbf{y}$, i.e., $\mathbf{x} \sqsubset \mathbf{p} \sqsubset \mathbf{y}$ for all $\mathbf{p} \in R$, we thus have $\mathbf{x} \sqsubset \wedge R$ and $\vee R \sqsubset \mathbf{y}$.

Before interpreting the majorization lattice, let us first introduce some notations that will be used frequently in this 
section:

$$
\begin{aligned}
\mathbb{R}_{+}^{d} & :=\left\{\mathbf{x} \in \mathbb{R}^{d} \| x_{k} \geqslant 0, \forall 1 \leqslant k \leqslant d\right\}, \\
\mathbb{R}_{+}^{d, \downarrow} & :=\left\{\mathbf{x} \in \mathbb{R}_{+}^{d} \| x_{k} \geqslant x_{k+1}, \forall 1 \leqslant k \leqslant d-1\right\}, \\
\mathbb{P}_{n}^{d} & :=\left\{\mathbf{x} \in \mathbb{R}_{+}^{d} \| \sum_{k} x_{k}=n\right\}, \\
\mathbb{P}_{n}^{d, \downarrow} & :=\mathbb{P}_{n}^{d} \cap \mathbb{R}_{+}^{d, \downarrow} .
\end{aligned}
$$

With these notations, we now introduce the relation between lattice and majorization, which was established by the notion of weak majorization in Bapat's work [47].

Definition 10 (Weak Majorization). For $\mathbf{x}=\left(x_{k}\right)_{k}, \mathbf{y}=$ $\left(y_{k}\right)_{k} \in \mathbb{R}^{d}$, we say that $\mathbf{x}$ is weakly majorized by $\mathbf{y}$, denoted by $\mathbf{x} \prec_{\mathrm{w}} \mathbf{y}$ if $\sum_{k=1}^{i} x_{k}^{\downarrow} \leqslant \sum_{k=1}^{i} y_{k}^{\downarrow}$ for all $1 \leqslant i \leqslant d$.

Due to the importance of the majorization lattice, we will review historical developments of this topic briefly. Some useful results will also be given in this section. During Bapat's investigations of the singular values of complex square matrices [47], the completeness of weak majorization on $\mathbb{R}_{+}^{d, \downarrow}$ was obtained as a byproduct.

Lemma 11 (Bapat). Let $S \subset \mathbb{R}_{+}^{d}$ be a nonempty set; then there exists a unique GLB, denoted by $\wedge S$, under weak majorization " $\prec_{\mathrm{w}}$ ".

Lemma 12 (Bapat). Let $S \subset \mathbb{R}_{+}^{d}$ be a bounded set, i.e., $\mathbf{x} \prec_{\mathrm{w}} S \prec_{\mathrm{w}} \mathbf{y}$ for some $\mathbf{x}$ and $\mathbf{y} \in \mathbb{R}_{+}^{d}$; then there exists a unique LUB, denoted by $\vee S$, under weak majorization " $\prec_{\mathrm{w}}$ ".

Then it can be shown that, for the set $\mathbb{P}_{n}^{d, \downarrow}$, the quadruple $\left(\mathbb{P}_{n}^{d, \downarrow}, \prec_{\mathrm{w}}, \wedge, \vee\right)$ is bounded since

$$
(n / d, \ldots, n / d) \prec_{\mathrm{w}} \mathbb{P}_{n}^{d, \downarrow} \prec_{\mathrm{w}}(n, 0, \ldots, 0),
$$

which immediately implies that, for any nonempty subset $S \subset$ $\mathbb{P}_{n}^{d, \downarrow} \subset \mathbb{R}_{+}^{d}$, it is bounded and has unique GLB $\wedge S$ and LUB $\vee S$. Thus, $\mathbb{P}_{n}^{d, \downarrow}$ is complete under “ $\prec_{\mathrm{w}}$ ".

Corollary 13. The quadruple $\left(\mathbb{P}_{n}^{d, \downarrow}, \prec_{\mathrm{w}}, \wedge, \vee\right)$ forms a complete lattice.

Here, we would like to note that, for the set $\mathbb{P}_{n}^{d}$, weak majorization " $\prec_{\mathrm{w}}$ " is only a preorder, i.e., a binary relation that is both reflexive and transitive. However, " $\prec_{\mathrm{w}}$ " is not antisymmetric; that is, we cannot obtain $\mathbf{x}=\mathbf{y}$ when $\mathbf{x} \prec_{\mathrm{w}} \mathbf{y}$ and $\mathbf{y} \prec_{\mathrm{w}} \mathbf{x}$ hold. For example, by taking $\mathbf{x}=(1,0)$ and $\mathbf{y}=$ $(0,1) \in \mathbb{P}_{1}^{d}$, we have $(1,0) \prec_{\mathrm{w}}(0,1)$ and $(0,1) \prec_{\mathrm{w}}(1,0)$, but $(1,0) \neq(0,1)$. Accordingly, $\left(\mathbb{P}_{n}^{d}, \prec_{\mathrm{w}}, \wedge, \vee\right)$ is not even a lattice. Weak majorization " $\prec_{\mathrm{w}}$ " becomes a partial order when all the probability distribution vectors are arranged in nonincreasing order, i.e., embedded into $\mathbb{P}_{n}^{d, \downarrow}$.

We now demonstrate that not only $\left(\mathbb{P}_{n}^{d, \downarrow}, \prec_{\mathrm{w}}, \wedge, \vee\right)$, but also $\left(\mathbb{P}_{n}^{d, \downarrow}, \prec, \wedge, \vee\right)$ with majorization " $\prec$ " forms a complete lattice. According to corollary 13, there exist the GLB $\wedge S$ and LUB $\vee S$ for any nonempty subset $S$ of $\mathbb{P}_{n}^{d, \downarrow}$, such that

$$
\wedge S \prec_{\mathrm{w}} S \prec_{\mathrm{w}} \vee S .
$$

By considering the trivial bounds of subset $S \subset \mathbb{P}_{n}^{d, \downarrow}$, i.e., $(n / d, \ldots, n / d),(n, 0, \ldots, 0) \in \mathbb{P}_{n}^{d, \downarrow}$, which satisfies $(n / d, \ldots, n / d) \prec_{\mathrm{w}} S \prec_{\mathrm{w}}(n, 0, \ldots, 0)$, we know that

$$
\begin{aligned}
& (n / d, \ldots, n / d) \prec_{\mathrm{w}} \wedge S \prec_{\mathrm{w}}(n, 0, \ldots, 0), \\
& (n / d, \ldots, n / d) \prec_{\mathrm{w}} \vee S \prec_{\mathrm{w}}(n, 0, \ldots, 0),
\end{aligned}
$$

which implies $\|\wedge S\|_{1}=\|\vee S\|_{1}=n$, and hence, $\wedge S \prec S \prec$ $\vee S$ holds for majorization " $\prec$ ". Until now, we have shown that $\wedge S$ and $\vee S$ are lower bound and upper bound for $S$, respectively. Now it is time to prove that they are optimal under majorization. For any vector $\mathbf{x} \prec S$, it is also a lower bound for weak majorization, i.e., $\mathbf{x} \prec_{\mathrm{w}} S$, and hence, $\mathbf{x} \prec_{\mathrm{w}} \wedge S$. Due to the fact that $\mathbf{x} \in \mathbb{P}_{n}^{d, \downarrow}$, we have $\|\mathbf{x}\|_{1}=\|\wedge S\|_{1}=n$, and thus, $\mathbf{x} \prec \wedge S$. Therefore, $\wedge S$ is the GLB for $S$ under majorization. Similarly, we have that $\vee S$ is the LUB for $S$ under majorization, which leads to the following statement:

Corollary 14. The quadruple $\left(\mathbb{P}_{n}^{d, \downarrow}, \prec, \wedge, \vee\right)$ forms a complete lattice.

A special class of corollary 14 is that $\left(\mathbb{P}_{1}^{d, \downarrow}, \prec, \wedge, \vee\right)$ forms a complete lattice, i.e., the probability simplex in finite dimensional space with nonincreasing order forms a complete lattice [46]. Moreover, this result has been used to derive the optimal common resource in majorization-based resource theories [49] and optimal direct-sum UURs for quantum states $[24,50]$ recently.

Now it is clear from the context that the optimal bound for $\mathbf{p} \otimes \mathbf{q}$ exists. Define the set $S_{\otimes}^{\mathrm{pre}}:=\{\mathbf{p} \otimes \mathbf{q}\}$, where $\mathbf{p}$ and $\mathbf{q}$ are obtained by performing process-channel measurements $\mathcal{T}_{1}$ and $\mathcal{T}_{2}$ to a quantum process, respectively. Then the set $S_{\otimes}:=S_{\otimes}^{\text {pre }} \cap \mathbb{P}_{1}^{d, \downarrow} \subset \mathbb{P}_{1}^{d, \downarrow}$, and our corollary 14 immediately implies the existence of $\wedge S_{\otimes}$ and $\vee S_{\otimes}$ under majorization:

$$
\wedge S_{\otimes} \prec \mathbf{p} \otimes \mathbf{q} \prec \vee S_{\otimes},
$$

Even though corollary 14 ensures the existence of both the upper and lower bounds of $\mathbf{p} \otimes \mathbf{q}$, it does not teach us how to find them effectively. Note also that the completeness of $\left(\mathbb{P}_{1}^{d, \downarrow}, \prec, \wedge, \vee\right)$ cannot be applied to the direct-sum form straightway since $\mathbf{p} \oplus \mathbf{q} \notin \mathbb{P}_{1}^{d, \downarrow}$. In this case, we can define the set $S_{\oplus}^{\text {pre }}:=\{\mathbf{p} \oplus \mathbf{q}\}$, and $S_{\oplus}:=S_{\oplus}^{\text {pre }} \cap \mathbb{P}_{2}^{d, \downarrow} \subset \mathbb{P}_{2}^{d, \downarrow}$. The existence of the GLB $\wedge S_{\oplus}$ and LUB $\vee S_{\oplus}$ is guaranteed by corollary 14 , which satisfies

$$
\wedge S_{\oplus} \prec \mathbf{p} \oplus \mathbf{q} \prec \vee S_{\oplus},
$$

with $\mathbf{p}$ and $\mathbf{q}$ obtained by performing process-channel measurements $\mathcal{T}_{1}$ and $\mathcal{T}_{2}$ to a quantum process, respectively.

To find the optimal bounds for $S_{\oplus}$, an additional process, namely flatness process, is needed. The lattice structure of majorization was revisited by Cicalese and Vaccaro in the study of its supermodularity and subadditivity properties [46], and the well-known flatness process $\mathcal{F}$ was introduced.

Definition 15 (Flatness Process). Let $\mathbf{x} \in \mathbb{R}_{+}^{d}$ be a vector and $j$ be the smallest integer in $\{2, \ldots, d\}$ such that $x_{j}>x_{j-1}$ and $i$ be the greatest integer in $\{1, \ldots, j-1\}$ such that $x_{i-1} \geqslant$ $\left(\sum_{k=i}^{j} x_{k}\right) /(j-i+1):=a$. Define

$$
\mathcal{T}(\mathbf{x}):=\left(x_{1}^{\prime}, \ldots, x_{n}^{\prime}\right) \text { with } x_{k}^{\prime}= \begin{cases}a & \text { for } k=i, \ldots, j \\ x_{k} & \text { otherwise. }\end{cases}
$$

and $\mathcal{F}(\mathbf{x}):=\mathcal{T}^{d-1}(\mathbf{x})=\mathcal{T}\left[\mathcal{T}^{d-2}(\mathbf{x})\right]$, i.e., applying $\mathcal{T}$ on the vector $\mathbf{x}$ successively $d-1$ times. Here, we call $\mathcal{F}$ the flatness process of vector $\mathbf{x}$, which satisfies the following lemma:

Lemma 16 (Cicalese-Vaccaro). For any $\mathbf{x} \in \mathbb{P}_{n}^{d}$, we have $\mathcal{F}(\mathbf{x}) \in \mathbb{P}_{n}^{d, \downarrow}$, and $\sum_{i=1}^{k} x_{i} \leqslant \sum_{i=1}^{k} x_{i}^{\prime}$ for all $1 \leqslant k \leqslant d$. 
Moreover, for all $\mathbf{y} \in \mathbb{P}_{n}^{d, \downarrow}$, we have

$$
\sum_{i=1}^{k} x_{i} \leqslant \sum_{i=1}^{k} y_{i}, \quad \forall 1 \leqslant k \leqslant d \Rightarrow \mathcal{F}(\mathbf{x}) \prec \mathbf{y} .
$$

We stress here that the original statement of flatness process $\mathcal{F}$, including its definition and lemma 16, introduced in Ref. [46] is only designed for the set $\mathbb{P}_{1}^{d}$, i.e., probability simplex. However, its generalization for vectors in $\mathbb{P}_{n}^{d}$, i.e., lemma 16, is also valid. The corresponding proof was given in our recent paper [24].

All these properties mentioned above lead to a standard approach in finding the optimal bounds for a subset $S$ of $\mathbb{P}_{n}^{d, \downarrow}$. Formally, let us consider $S \subset \mathbb{P}_{n}^{d, \downarrow}$, and then there are two steps in constructing its GLB $\wedge S$ and LUB $\vee S$. The first step is to find the quantities $a_{k}$ and $b_{k}$, which are defined as

$$
\begin{aligned}
a_{k} & :=\left(\min _{\mathbf{x} \in S} \sum_{i=1}^{k} x_{i}\right)-\sum_{i=1}^{k-1} a_{i}, \\
b_{k} & :=\left(\max _{\mathbf{x} \in S} \sum_{i=1}^{k} x_{i}\right)-\sum_{i=1}^{k-1} b_{i},
\end{aligned}
$$

for $1 \leqslant k \leqslant d$. It is immediate to observe that the vector $\mathbf{a}_{S}:=$ $\left(a_{k}\right)_{k} \in \mathbb{P}_{n}^{d, \downarrow}$. On the other hand, the vector $\mathbf{b}_{S}:=\left(b_{k}\right)_{k}$ might not always belong to the set $\mathbb{P}_{n}^{d, \downarrow}$. To give our reader some intuition, we recall the example constructed in Ref. [46].

Example 17. Take $S=\{\mathbf{x}, \mathbf{y}\}$ with

$$
\begin{aligned}
& \mathbf{x}=(0.6,0.15,0.15,0.1) \\
& \mathbf{y}=(0.5,0.25,0.2,0.05)
\end{aligned}
$$

Then in this case, $\mathbf{b}_{S}=(0.6,0.15,0.2,0.05)$, which does not belong to the set $\mathbb{P}_{1}^{d, \downarrow}$ since $b_{2}=0.15<b_{3}=0.2$. Even though we rearrange the vector $\mathbf{b}_{S}$ into nonincreasing or$\operatorname{der} \mathbf{b}_{S}^{\downarrow}=(0.6,0.2,0.15,0.05), \mathbf{b}_{S}^{\downarrow}$ is not the optimal upper bound, i.e., $\mathbf{b}_{S}^{\downarrow} \neq \vee S$ (see Fig. 2), since in this case,

$$
\vee S=\mathbf{x} \vee \mathbf{y}=\mathcal{F}\left(\mathbf{b}_{S}\right)=(0.6,0.175,0.175,0.05) .
$$

In general, the second step in constructing the optimal bounds for $S$ with majorization is to keep $\mathbf{a}_{S}$ fixed and apply the flatness process $\mathcal{F}$ to $\mathbf{b}_{S}$. Formally, our corollary 14 and lemma 16 imply the optimality of $\mathbf{a}_{S}$ and $\mathcal{F}\left(\mathbf{b}_{S}\right)$ :

Corollary 18. For any nonempty subset $S \subset \mathbb{P}_{n}^{d, \downarrow}$, its GLB $\wedge S$ and LUB $\vee S$ under majorization are given by

$$
\wedge S=\mathbf{a}_{S}, \quad \vee S=\mathcal{F}\left(\mathbf{b}_{S}\right),
$$

where $\mathcal{F}$ stands for the flatness process defined in definition 15 , and $\mathbf{a}_{S}, \mathbf{b}_{S}$ are defined in Eq. (C10).

Proof. Here, the existence of $\wedge S$ and $\vee S$ for $S$ are guaranteed by corollary 14 . We first prove $\wedge S=\mathbf{a}_{S}$. By hypothesis, for any vector $\mathbf{c} \in \mathbb{P}_{n}^{d, \downarrow}$ such that

$$
\mathbf{c} \prec S,
$$

we have

$$
\sum_{i=1}^{k} c_{i} \leqslant \sum_{i=1}^{k} a_{i}
$$

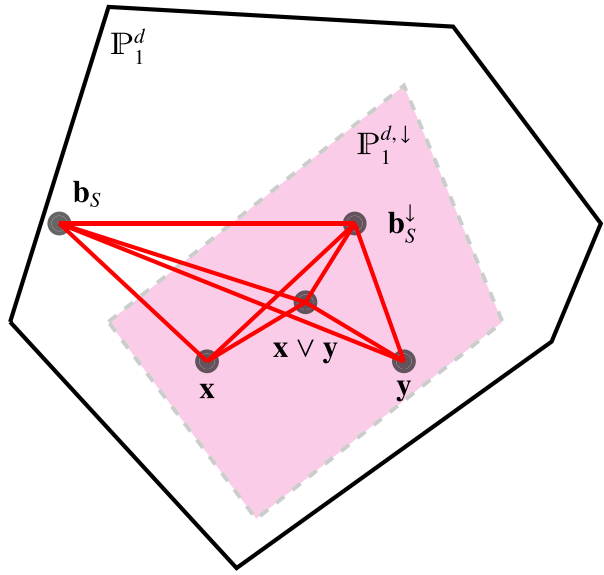

FIG. 2. Schematic illustration of the lattice structure exhibited in example 17 excluding the GLB. Each point stands for an element, and the red line represents the binary relation " $\prec$ " between elements. In this plot, a lower point is majorized by the higher point whenever they are connected with a red line. Obviously, here, $\mathbf{b}_{S}^{\downarrow} \prec \mathbf{b}_{S}$ and $\mathbf{b}_{S} \prec \mathbf{b}_{S}^{\downarrow}$, but $\mathbf{b}_{S} \neq \mathbf{b}_{S}^{\downarrow}$.

for all $1 \leqslant k \leqslant d$, and thus,

$$
\mathbf{c} \prec \mathbf{a}_{S} .
$$

By choosing $\mathbf{c}$ as $\wedge S$, we obtain $\wedge S \prec \mathbf{a}_{S}$. By the definition of $\mathbf{a}_{S}$, we have $\mathbf{a}_{S} \prec S$, and hence, $\mathbf{a}_{S} \prec \wedge S$. Thus, $\wedge S=\mathbf{a}_{S}$. The equation holds since majorization has the property of antisymmetricity on $\mathbb{P}_{n}^{d, \downarrow}$, with both $\mathbf{a}_{S}$ and $\wedge S$ belonging to the set $\mathbb{P}_{n}^{d, \downarrow}$.

Next, we move on to show $\vee S=\mathcal{F}\left(\mathbf{b}_{S}\right)$. By hypothesis, for any vector $\mathbf{d} \in \mathbb{P}_{n}^{d, \downarrow}$ such that

$$
S \prec \mathbf{d} \text {, }
$$

we have

$$
\sum_{i=1}^{k} b_{i} \leqslant \sum_{i=1}^{k} d_{i}
$$

for all $1 \leqslant k \leqslant d$. Now by using lemma 16 directly, we get

$$
\mathcal{F}\left(\mathbf{b}_{S}\right) \prec \mathbf{d},
$$

as expected. Note that $\mathcal{F}\left(\mathbf{b}_{S}\right) \prec \mathbf{b}_{S}$ since $\sum_{i=1}^{k} b_{i} \leqslant \sum_{i=1}^{k} b_{i}$. By choosing $\mathbf{d}$ as $\vee S$, we obtain $\mathcal{F}\left(\mathbf{b}_{S}\right) \prec \vee S$. By using the fact that $\sum_{i=1}^{k} x_{i} \leqslant \sum_{i=1}^{k} x_{i}^{\prime}$ for all $1 \leqslant k \leqslant d$, and $\mathbf{x} \in S$, we have $S \prec \mathcal{F}\left(\mathbf{b}_{S}\right)$, and hence, $\vee S \prec \mathcal{F}\left(\mathbf{b}_{S}\right)$. Thus, $\vee S=$ $\mathcal{F}\left(\mathbf{b}_{S}\right)$. The equation holds since majorization has the property of antisymmetricity on $\mathbb{P}_{n}^{d, \downarrow}$, with both $\mathcal{F}\left(\mathbf{b}_{S}\right)$ and $\vee S$ belonging to the set $\mathbb{P}_{n}^{d, \downarrow}$.

As an application of our corollary 18 , take $S$ as $S_{\oplus}^{\downarrow} \subset \mathbb{P}_{2}^{d, \downarrow}$, which immediately yields $\mathbf{b}_{S_{\oplus}^{\downarrow}}=\mathbf{s}$ defined in Eq. (12) from our main text. Therefore, $\mathcal{F}(\mathbf{s})=\mathcal{F}\left(\mathbf{b}_{S_{\oplus}^{\downarrow}}\right)=\vee S_{\oplus}^{\downarrow}$ is the optimal upper bound for UURs for all quantum processes in the form of direct sum. Formally,

Corollary 19. For probability vectors $\mathbf{p}$ and $\mathbf{q}$ obtained by measuring $\Psi$ with respect to $\mathcal{T}_{1}:=\left(\rho^{R A}, M\right)$ and $\mathcal{T}_{2}:=$ $\left(\sigma^{R A}, N\right)$, their joint uncertainties in terms of $\mathbf{p} \oplus \mathbf{q}$ is therefore bounded by a vector independent of quantum process $\Psi$ of the form

$$
\mathbf{p} \oplus \mathbf{q} \prec \mathcal{F}(\mathbf{s})=\mathcal{F}\left(s_{1}, s_{2}-s_{1}, s_{3}-s_{2}, \ldots, 0\right) .
$$


Here, $\mathcal{F}$ is the flatness process defined in definition $15, \mathcal{F}(\mathbf{s})$ is the optimal bound for $\mathbf{p} \oplus \mathbf{q}$, and each $s_{k}$ is a functional of the conditional min-entropy

$$
s_{k}:=\max _{I_{k}} 2^{-\mathrm{H}_{\min }(B \mid A)_{G\left(I_{k}\right)}},
$$

where the maximum is over all subset $\mathcal{I}_{k}$, and the conditional min-entropy for $G\left(\mathcal{I}_{k}\right)$ is defined as

$$
\mathrm{H}_{\min }(B \mid A)_{G\left(I_{k}\right)}:=-\log \inf _{X^{A} \geqslant 0}\left\{\operatorname{Tr}\left(X^{A}\right) \mid X^{A} \otimes \mathbb{1}^{B} \geqslant G\left(\mathcal{I}_{k}\right)\right\} .
$$

It turns out that not only the optimal upper bound $\mathcal{F}\left(\mathbf{b}_{S_{\oplus}^{\downarrow}}\right)$ of $S_{\oplus}^{\downarrow} \subset \mathbb{P}_{2}^{d, \downarrow}$, i.e., direct-sum UURs for quantum processes, can be evaluated explicitly by the means of SDP and flatness process, but also the optimal lower bound $\mathbf{a}_{S_{\oplus}^{\downarrow}}$ of the reverse direct-sum UURs for quantum processes.

\section{APPENDIX D: PROOF OF THEOREM 4}

In this section, we turn our attention back to the UURs for quantum processes in the form of direct product. We first consider the sum of the first $k$ largest components of $\mathbf{p} \otimes \mathbf{q}$, i.e.,

$$
\begin{aligned}
& \max _{T_{k}} \max _{\Psi}\left(\sum_{(x, y) \in T_{k}} p_{x} q_{y}\right) \\
& \leqslant \max _{|R|+|S|=k+1} \max _{\Psi}\left(\frac{\sum_{x \in R} p_{x}+\sum_{y \in S} q_{y}}{2}\right)^{2} \\
& =\max _{I_{k+1}} \max _{\Psi}\left(\frac{\operatorname{Tr}\left[G\left(I_{k+1}\right) J_{\Psi}^{A B}\right]}{2}\right)^{2}=\left(\frac{s_{k+1}}{2}\right)^{2}=t_{k},
\end{aligned}
$$

where the outer maximum is over all subsets $T_{k} \subset[\mathrm{m}] \times$ $[n]$ such that $\left|T_{k}\right|=k$, with $[m]:=\{1, \ldots, m\}$ and $[n]:=$ $\{1, \ldots, n\}$. Therefore, $\mathbf{t}$ provides an upper bound of UURs for quantum processes, which completes the proof of our theorem 4.

Moreover, by definition of $S_{\otimes}^{\downarrow} \subset \mathbb{P}_{1}^{d, \downarrow}$, and the iterated application of corollary 18 , we have that

$$
\mathbf{a}_{S_{\otimes}^{\downarrow}} \prec \mathbf{p} \otimes \mathbf{q} \prec \mathcal{F}\left(\mathbf{b}_{S_{\otimes}^{\downarrow}}\right)=\vee S_{\otimes}^{\downarrow} .
$$

It holds also that

$$
\mathcal{F}\left(\mathbf{b}_{S_{\otimes}^{\downarrow}}\right) \prec \mathbf{b}_{S_{\otimes}^{\downarrow}} .
$$

Hence, the bounds for $\mathbf{p} \otimes \mathbf{q}$ can be ordered as

$$
\mathbf{a}_{S_{\otimes}^{\downarrow}} \prec \mathbf{p} \otimes \mathbf{q} \prec \mathcal{F}\left(\mathbf{b}_{S_{\otimes}^{\downarrow}}\right) \prec \mathbf{b}_{S_{\otimes}^{\downarrow}} .
$$

From Eq. (D1), it turns out that the bound $\mathbf{b}_{S_{\otimes}^{\downarrow}}$ is majorized by the one constructed in our main text, that is, $\mathbf{b}_{S_{\otimes}^{\downarrow}} \prec \mathbf{t}$. Note that the quantity $\max _{T_{k}} \max _{\Psi}\left(\sum_{(x, y) \in T_{k}} p_{x} q_{y}\right)$ is exactly the sum of the first $k$ largest components of $\mathbf{b}_{S_{\circledast}^{\downarrow}}$, and usually,

$$
\mathbf{b}_{S_{\otimes}^{\downarrow}} \neq \mathbf{b}_{S_{\otimes}^{\downarrow}}^{\downarrow},
$$

i.e., $\mathbf{b}_{S_{\otimes}^{\downarrow}} \notin \mathbb{P}_{1}^{d, \downarrow}$. Similarly, we have $\mathbf{t} \neq \mathbf{t}^{\downarrow}$ in general, and hence, $\mathbf{t} \notin \mathbb{P}_{1}^{d, \downarrow}$ does not hold in general.

It is interesting to identify the sum of the first $k$ largest components of $\mathcal{F}(\mathbf{t})$. Let us denote the $i$ th element of $\mathcal{F}(\mathbf{t})$ as
$[\mathcal{F}(\mathbf{t})]_{i}$. Then we have

$$
\max _{T_{k}} \max _{\Psi}\left(\sum_{(x, y) \in T_{k}} p_{x} q_{y}\right) \leqslant t_{k} \leqslant \sum_{i=1}^{k}[\mathcal{F}(\mathbf{t})]_{i},
$$

and hence, from lemma 16, we arrive at the following expression:

$$
\mathbf{a}_{S_{\otimes}^{\downarrow}} \prec \mathbf{p} \otimes \mathbf{q} \prec \mathcal{F}\left(\mathbf{b}_{S_{\circledast}^{\downarrow}}\right) \prec \mathcal{F}(\mathbf{t}) \prec \mathbf{t} .
$$

If the quantum processes considered here are state-preparation channel, then this chain of bounds makes an improvement over previous results of UURs introduced in Ref. [23] since $\mathcal{F}(\mathbf{t}) \prec \mathbf{t}$. As a byproduct, the optimal bound $\mathbf{a}_{S_{\otimes}^{\downarrow}}$ of the reverse direct-product UURs for quantum processes is also given.

\section{APPENDIX E: FOLLOW-UP RESEARCH DIRECTIONS}

There are plenty of important directions of investigations which we leave for future work. First of all, we did not explore here the extension of our results to the cases with bipartite quantum channels [54,55], where the measured quantum channel is prepared entangled with another channel, a dynamic quantum memory that might be possible to predict the outcomes for both process-channel measurements $\mathcal{T}_{1}, \mathcal{T}_{2}$ simultaneously, which is the generalized uncertainty principle in the presence of dynamic quantum memory [2]. It would also be interesting to study how the use of dynamic quantum memory can further strengthen the power of quantum cryptography.

Another important direction of investigation is the noise and disturbance tradeoff in process-channel measurements [56]. To capture the idea of "how accurate" a process-channel measurement $\mathcal{T}_{1}$ is, we should consider its measuring apparatus $\mathscr{T}$, and the corresponding error $\mathscr{E}\left(\mathcal{T}_{1}, \mathscr{T}\right)$, or noise, which is quantifies through operational measurement statistics. When the measured channel is subjected to the apparatus $\mathscr{T}$, another process-channel measurement $\mathcal{T}_{2}$ will be disturbed and lead to the disturbance $\mathscr{D}\left(\mathcal{T}_{2}, \mathscr{T}\right)$. The aim of this direction of investigation is to introduce the operational definitions for $\mathscr{E}$ and $\mathscr{D}$ such that $\mathscr{E}\left(\mathcal{T}_{1}, \mathscr{T}\right)+\mathscr{D}\left(\mathcal{T}_{2}, \mathscr{T}\right) \geqslant$ $-2 \log c\left(\mathcal{T}_{1}, \mathcal{T}_{2}\right)$.

Finally, when considering the process-channel measurements with possibilities of small errors, we should employ smooth entropies to obtain meaningful results. Therefore, it would be important to generalize our entropic uncertainty relation for quantum processes to the one expressed in terms of smooth entropies [57]. Nevertheless, these generalizations are nontrivial and are left for future work.

\section{APPENDIX F: CONJECTURE}

In this section, we give a conjecture on the Shannon entropic uncertainty relation for quantum processes. Given two process-channel measurements $\mathcal{T}_{1}$ and $\mathcal{T}_{2}$, their overlaps are defined by $c_{x y}\left(\mathcal{T}_{1}, \mathcal{T}_{2}\right):=\left\|\tilde{E}_{x}^{1 / 2} \tilde{F}_{y}^{1 / 2}\right\|$ with $1 \leqslant$ $x \leqslant m+1$ and $1 \leqslant y \leqslant n+1$, and the entropic uncertainty relations in the form of $\mathrm{H}_{\alpha}\left(\mathcal{T}_{1}\right)+\mathrm{H}_{\beta}\left(\mathcal{T}_{2}\right)$, with $1 / \alpha+1 / \beta=2$, is lower-bounded by $-2 \log c\left(\mathcal{T}_{1}, \mathcal{T}_{2}\right)=$ $-2 \log \max _{x, y} c\left(\mathcal{T}_{1}, \mathcal{T}_{2}\right)$, which is shown in our main text. 
This bound is tight for the case with $1 / \alpha+1 / \beta=2$. However, we do not know whether this is also tight for the case with $\alpha=\beta=1$.

As a matter of convenience, let us rearrange the overlaps between $\mathcal{T}_{1}$ and $\mathcal{T}_{2}$ in nonincreasing order and denote the $k$ largest overlap as $c_{k}\left(\mathcal{T}_{1}, \mathcal{T}_{2}\right)$; then $c\left(\mathcal{T}_{1}, \mathcal{T}_{2}\right)=c_{1}\left(\mathcal{T}_{1}, \mathcal{T}_{2}\right)$. Now we have a chain of overlaps

$$
c_{1}\left(\mathcal{T}_{1}, \mathcal{T}_{2}\right) \geqslant c_{2}\left(\mathcal{T}_{1}, \mathcal{T}_{2}\right) \geqslant \cdots \geqslant c_{(m+1)(n+1)}\left(\mathcal{T}_{1}, \mathcal{T}_{2}\right),
$$

and we would like to know whether the Shannon entropic uncertainty relation can be further improved to

$$
\begin{gathered}
\mathrm{H}\left(\mathcal{T}_{1}\right)+\mathrm{H}\left(\mathcal{T}_{2}\right) \geqslant-2 \log c_{1}\left(\mathcal{T}_{1}, \mathcal{T}_{2}\right) \\
+\sum_{k}\left(2-s_{2 k}\right) \log \frac{c_{k}\left(\mathcal{T}_{1}, \mathcal{T}_{2}\right)}{c_{k+1}\left(\mathcal{T}_{1}, \mathcal{T}_{2}\right)},
\end{gathered}
$$

with $s_{k}$ as defined in Eq. (13) of our main text. In fact, when the object of our study is state-preparation channel, then the validity of above entropic uncertainty relation is proved by replacing $c_{k}\left(\mathcal{T}_{1}, \mathcal{T}_{2}\right)$ with $c_{k}(M, N)$ in Ref. [58].

We will finish by expounding the motivations of this conjecture. Firstly, the process-independent bound depends only on the process-channel measurements $\mathcal{T}_{1}$ and $\mathcal{T}_{2}$ and hence quantify the intrinsic incompatibility between them. However, in the context of incompatibility, the process-independent bound is by no means only dependent on the largest overlap between $\mathcal{T}_{1}$ and $\mathcal{T}_{2}$, but not all overlaps. The incompatibility between them should be completely characterized by the set of all overlaps. Secondly, it is worth noting that the bound of entropic uncertainty relation could be directly used to prove cryptography security [59].
[1] W. Heisenberg, Über den anschaulichen inhalt der quantentheoretischen kinematik und mechanik, Z. Phys. 43, 172 (1927).

[2] M. Berta, M. Christandl, R. Colbeck, J. M. Renes, and R. Renner, The uncertainty principle in the presence of quantum memory, Nat. Phys. 6, 659 (2010).

[3] E. Chitambar and G. Gour, Quantum resource theories, Rev. Mod. Phys. 91, 025001 (2019).

[4] H. F. Hofmann and S. Takeuchi, Violation of local uncertainty relations as a signature of entanglement, Phys. Rev. A 68, 032103 (2003).

[5] H. F. Hofmann, Bound entangled states violate a nonsymmetric local uncertainty relation, Phys. Rev. A 68, 034307 (2003).

[6] O. Gühne, Characterizing Entanglement Via Uncertainty Relations, Phys. Rev. Lett. 92, 117903 (2004).

[7] O. Gühne and M. Lewenstein, Entropic uncertainty relations and entanglement, Phys. Rev. A 70, 022316 (2004).

[8] Y. Huang, Entanglement criteria via concave-function uncertainty relations, Phys. Rev. A 82, 012335 (2010).

[9] R. Schwonnek, L. Dammeier, and R. F. Werner, StateIndependent Uncertainty Relations and Entanglement Detection in Noisy Systems, Phys. Rev. Lett. 119, 170404 (2017).

[10] Y.-Y. Zhao, G.-Y. Xiang, X.-M. Hu, B.-H. Liu, C.-F. Li, G.-C. Guo, R. Schwonnek, and R. Wolf, Entanglement Detection by Violations of Noisy Uncertainty Relations: A Proof of Principle, Phys. Rev. Lett. 122, 220401 (2019).

[11] M. D. Reid, Demonstration of the Einstein-Podolsky-Rosen paradox using nondegenerate parametric amplification, Phys. Rev. A 40, 913 (1989).

[12] J. Schneeloch, C. J. Broadbent, S. P. Walborn, E. G. Cavalcanti, and J. C. Howell, Einstein-Podolsky-Rosen steering inequalities from entropic uncertainty relations, Phys. Rev. A 87, 062103 (2013).

[13] A. Rutkowski, A. Buraczewski, P. Horodecki, and M. Stobińska, Quantum Steering Inequality with Tolerance for Measurement-Setting Errors: Experimentally Feasible Signature of Unbounded Violation, Phys. Rev. Lett. 118, 020402 (2017).

[14] A. Riccardi, C. Macchiavello, and L. Maccone, Multipartite steering inequalities based on entropic uncertainty relations, Phys. Rev. A 97, 052307 (2018).

[15] Y. Xiao, Y. Xiang, Q. He, and B. C. Sanders, Quasi-fine-grained uncertainty relations, New J. Phys. 22, 073063 (2020).
[16] A. C. S. Costa, R. Uola, and O. Gühne, Steering criteria from general entropic uncertainty relations, Phys. Rev. A 98, 050104(R) (2018).

[17] R. Uola, A. C. S. Costa, H. C. Nguyen, and O. Gühne, Quantum steering, Rev. Mod. Phys. 92, 015001 (2020).

[18] J. Oppenheim and S. Wehner, The uncertainty principle determines the nonlocality of quantum mechanics, Science $\mathbf{3 3 0}$, 1072 (2010).

[19] E. H. Kennard, Zur quantenmechanik einfacher bewegungstypen, Z. Phys. 44, 326 (1927).

[20] H. Weyl, Gruppentheorie und Quantenmechanik. (Hirzel, Leipzig, 1928), VIII, $288 \mathrm{~S}$.

[21] H. Maassen and J. B. M. Uffink, Generalized Entropic Uncertainty Relations, Phys. Rev. Lett. 60, 1103 (1988).

[22] G. H. Hardy, J. E. Littlewood, and G. Pólya, Inequalities (Cambridge University Press, Cambridge, 1952).

[23] S. Friedland, V. Gheorghiu, and G. Gour, Universal Uncertainty Relations, Phys. Rev. Lett. 111, 230401 (2013).

[24] Y. Yuan, Y. Xiao, Z. Hou, S.-M. Fei, G. Gour, G.-Y. Xiang, C.-F. $\mathrm{Li}$, and G.-C. Guo, Strong majorization uncertainty relations: theory and experiment (2019), arXiv:1912.13383 [quant-ph].

[25] Ł. Rudnicki, Z. Puchała, and K. Życzkowski, Majorization entropic uncertainty relations, J. Phys. A: Math. Theor. 46, 272002 (2013).

[26] Ł. Rudnicki, Z. Puchała, and K. Życzkowski, Strong majorization entropic uncertainty relations, Phys. Rev. A 89, 052115 (2014).

[27] A. Nitzan and M. A. Ratner, Electron transport in molecular wire junctions, Science 300, 1384 (2003).

[28] N. Lambert, Y.-N. Chen, Y.-C. Cheng, C.-M. Li, G.-Y. Chen, and F. Nori, Quantum biology, Nat. Phys. 9, 10 (2013).

[29] F. A. Pollock, C. Rodríguez-Rosario, T. Frauenheim, M. Paternostro, and K. Modi, Non-Markovian quantum processes: complete framework and efficient characterization, Phys. Rev. A 97, 012127 (2018).

[30] F. A. Pollock, C. Rodríguez-Rosario, T. Frauenheim, M. Paternostro, and K. Modi, Operational Markov Condition for Quantum Processes, Phys. Rev. Lett. 120, 040405 (2018).

[31] P. Taranto, F. A. Pollock, S. Milz, M. Tomamichel, and K. Modi, Quantum Markov Order, Phys. Rev. Lett. 122, 140401 (2019). 
[32] J. Biamonte, P. Wittek, N. Pancotti, P. Rebentrost, N. Wiebe, and S. Lloyd, Quantum machine learning, Nature 549, 195 (2017).

[33] A. Jamiołlkowski, Linear transformations which preserve trace and positive semidefiniteness of operators, Rep. Math. Phys. 3, 275 (1972).

[34] M.-D. Choi, Completely positive linear maps on complex matrices, Linear Algebra Its Appl. 10, 285 (1975).

[35] M. A. Nielsen and I. L. Chuang, Quantum Computation and Quantum Information: 10th Anniversary Edition (Cambridge University Press, Cambridge, 2010).

[36] M. M. Wilde, Quantum Information Theory, 2nd ed. (Cambridge University Press, Cambridge, 2017).

[37] J. Watrous, The Theory of Quantum Information (Cambridge University Press, Cambridge, 2018).

[38] M. Ziman, Process positive-operator-valued measure: a mathematical framework for the description of process tomography experiments, Phys. Rev. A 77, 062112 (2008).

[39] G. Chiribella, G. M. D’Ariano, and P. Perinotti, Transforming quantum operations: quantum supermaps, Europhys. Lett. 83, 30004 (2008).

[40] G. Chiribella, G. M. D’Ariano, and P. Perinotti, Quantum Circuit Architecture, Phys. Rev. Lett. 101, 060401 (2008).

[41] G. Gour, Comparison of quantum channels by superchannels, IEEE Trans. Inf. Theory 65, 5880 (2019).

[42] D. Deutsch, Uncertainty in Quantum Measurements, Phys. Rev. Lett. 50, 631 (1983).

[43] L. Gao, M. Junge, and N. LaRacuente, Uncertainty principle for quantum channels, 2018 IEEE International Symposium on Information Theory (ISIT), Vail, CO (IEEE, Piscataway, NJ, 2018), p. 996.

[44] A. E. Rastegin, Rényi formulation of the entropic uncertainty principle for POVMs, J. Phys. A: Math. Theor. 43, 155302 (2010).

[45] Y. Xiao, K. Fang, and G. Gour, The complementary information principle of quantum mechanics (2019), arXiv:1908.07694 [quant-ph].
[46] F. Cicalese and U. Vaccaro, Supermodularity and subadditivity properties of the entropy on the majorization lattice, IEEE Trans. Inf. Theory 48, 933 (2002).

[47] R. B. Bapat, Majorization and singular values. III, Linear Algebra Its Appl. 145, 59 (1991).

[48] J. V. Bondar, Inequalities: theory of majorization and its applications: by Albert W. Marshall and Ingram Olkin, Linear Algebra Its Appl. 199, 115 (1994).

[49] G. M. Bosyk, G. Bellomo, F. Holik, H. Freytes, and G. Sergioli, Optimal common resource in majorization-based resource theories, New J. Phys. 21, 083028 (2019).

[50] J.-L. Li and C.-F. Qiao, The optimal uncertainty relation, Ann. Phys. 531, 1900143 (2019).

[51] K. Kraus, Complementary observables and uncertainty relations, Phys. Rev. D 35, 3070 (1987).

[52] V. Paulsen, Completely Bounded Maps and Operator Algebras (Cambridge University Press, Cambridge, 2003).

[53] R. Renner, Security of quantum key distribution (2005), arXiv:quant-ph/0512258 [quant-ph].

[54] G. Gour and C. M. Scandolo, The entanglement of a bipartite channel (2019), arXiv:1907.02552 [quant-ph].

[55] S. Bäuml, S. Das, X. Wang, and M. M. Wilde, Resource theory of entanglement for bipartite quantum channels (2019), arXiv:1907.04181 [quant-ph].

[56] F. Buscemi, M. J. W. Hall, M. Ozawa, and M. M. Wilde, Noise and Disturbance in Quantum Measurements: An Information-Theoretic Approach, Phys. Rev. Lett. 112, 050401 (2014).

[57] M. Tomamichel and R. Renner, Uncertainty Relation for Smooth Entropies, Phys. Rev. Lett. 106, 110506 (2011).

[58] Y. Xiao, N. Jing, S.-M. Fei, and X. Li-Jost, Improved uncertainty relation in the presence of quantum memory, J. Phys. A: Math. Theor. 49, 49LT01 (2016).

[59] P. J. Coles, M. Berta, M. Tomamichel, and S. Wehner, Entropic uncertainty relations and their applications, Rev. Mod. Phys. 89, 015002 (2017). 